Www.jmscr.igmpublication.org

Impact Factor (SJIF): 6.379

Index Copernicus Value: 79.54

ISSN (e)-2347-176x ISSN (p) 2455-0450

crossrefDOI: https://dx.doi.org/10.18535/jmscr/v6i12.35

Journal Of Medical Science And Clinical Research

IGM Publication

An Official Publication of IGM Publication

\title{
Allogenic Blood Transfusion and Post-operative Outcomes in Elective CABG cases: A Prospective Study
}

\author{
Authors \\ Jain Pramesh ${ }^{1}$, Gupta Animesh ${ }^{2}$, Dr Ansuman Mondal ${ }^{3}$ \\ ${ }^{1}$ Postgraduate Resident, Department of CTVS, I.P.G.M.E \& R.SSKM Hospital, Kolkata- WB \\ ${ }^{2}$ Postgraduate Resident, Department of Cardiology, SJIC \& R, Bangalore - Karanataka \\ ${ }^{3}$ Associate Professor, Department of CTVS, I.P.G.M.E \& R.SSKM Hospital, Kolkata- WB
}

\begin{abstract}
Aims and Objectives: To assess the relationship between RBC/whole blood transfusion and hospital length of stay in a large, single reference centre of cardiac surgery and then to compare the characteristics of patients who received $R B C /$ whole blood transfusion with those who did not.

Material and Methods: The study will be conducted in the Dept of CTVS of SSKM Hospital, Kolkata-WB. Patients admitted for OP CABG intervention in CTVS department from April 2014 to July 2015. Sample size was 60 cardiac patients. Of the 60 patients included in analysis patients receiving blood transfusion are selected for study group. The control group consists of patients will not require blood transfusion.

Results: From the total of 126 patients randomized chose in this study.33 did not receive any RBC transfusion (26.19\%) labelled them Group 1; 55 (43.65\%) patients were given 1-3 units labelled them Group 2 and 38 (30.15\%) received more than 3 RBC units labelled them Group 3 in the first 72 postoperative hours. From 126 patients, 91 (72.22\%) patients received transfusion at the operative room and day O(day of surgery), 58 (46.03\%) received at the first postoperative day (1PO), 19(15.08\%) at the second postoperative day (2PO) and 5 (3.97\%) at the third postoperative day (3PO). Analysing postoperative clinical complications, We found that patients from the high transfusion group presented a higher incidence of postoperative complications related to the composite endpoint 30-day all-cause mortality $(p<0.017)$ and acute respiratory distress syndrome, and higher Re-intubation rates $(p<0.004)$. Regarding infections in patients from group 2 and group3 as compared with groupl we found that both groups were associated with significant $p$ values $\{$ (both deep and sternal infections) and ( $p<0.005 \&<0.020$ respectively)\} It indicate blood transfusion is independent morbid factor in OPCABG patients.

In our study, we compared ventilator weaning off time of the patients in different groups; we found that patients with blood transfused groups require much more time as compared to non-transfused group in all sub groups. In groupl 27(81.82\%) patients were extubated in 12-18 hrs postoperatively, and remaining 6 (18.18\%) patients in 18-24hrs postoperatively. In group2 41 (74.55\%) patients extubated in 12-18hrs, 11(20\%) patient's extubated in 18-24hrs, and only one pt took long time for weaning off from ventilator postoperatively. While in group3 only 11 (28.95\%) patients wean-off in 12-18 hrs, 19 (50\%) patients require 18-24 hrs time for extubation, and remaining 7 patients were extubated between 24-48 hrs postoperatively. 3 patients could not be taken off from ventilator. Higher mortality rates were directly related to blood transfusions. In our study total no. mortality was 10. Out of these 7 deaths occur in high transfusion group i.e. group3, 2 deaths occurred in group2 and one mortality had occurred in nontransfusedgroup. Main
\end{abstract}


objective our study was to found association between blood transfusion and length of stay (LOS-no. of days from day of surgery till discharge/death). In our study, Length of stay had range between 6-12 days in group 1; 6-28 days in group2, and 9-45 days in group3 with mean \& standard deviation of $7.71+/-1.67$ days in group1, 9.49 +/- 4.33 days in group2 and 16.18 +/- 7.64days in group3 respectively. When we were comparing $p$ values of groupl, $2 \& 3$ we found highly significant values among the groups. Compare the groupl \& 2 p value was 0.029; between group1 \&3 and Group $\& 3$ it was highly significant (0.0001). These calculations showed that no. of blood transfusions are directly responsible for increase in the length of hospitalisation of patient.

Conclusion: In conclusion, our data for OPCABG shows that patients who received transfusion required significantly longer ventilator support time, longer inotropic support time, increase rates of re-intubation. All these factors combine to increase morbidity and delays patient's recovery process. In our study, we found that blood transfusions increase the rates of superficial and deep sternal wound infections, increases hospital stay of patient. All above factors contributing to increase mortality in higher blood transfused patients. They also had significantly higher incidence of the 30-day mortality. Thus we suggest that for better patient outcome, early recovery, full utilisation of scarce resources, decrease unnecessary economic burden to community, we should approach toward the restricted blood transfusions and pre-op management of risk factors.

\section{Introduction}

Bleeding during and after cardiac operations and the hemodilution effects of cardiopulmonary bypass commonly result in blood transfusions. Red blood cell transfusion can both benefit and harm. Transfusion of allogenic red blood cells (RBCs)/whole blood is increasingly recognized as a risk factor for adverse outcome after cardiac surgery and blood transfusions during or after coronary artery bypass operations were associated with increased long-term mortality ${ }^{1}$.Unnecessary transfusions are likely to be associated with unnecessary morbidity and additional indirect hospitalization $\operatorname{cost}^{1}$. A low hematocrit in the absence of hemorrhagic shock remains the most common indication in the critically ill. The rationale for perioperative red blood cell (RBC)/ whole blood transfusion is based on the observations that anaemia is an independent risk factor for morbidity and mortality after cardiac surgery and that red blood cell/whole blood transfusion would benefit a subset of patients presenting tissue hypoperfusion ${ }^{2}$. Intra-operative transfusion of 1 unit to 2 units packed red blood cells/ whole blood is associated with increased 30day mortality, surgical-site infection, pneumonia, and sepsis in surgical patients ${ }^{3}$. The anemia and transfusion interaction was associated with an increased hazard of late mortality. Exposure to 1 or 2 units of RBCs/ whole blood was associated with a $16 \%$ increased hazard of decreased survival after cardiac surgery ${ }^{4}$. Exposure to 1 or 2 units of RBCs/ whole blood was associated with a $16 \%$ increased hazard of decreased survival after cardiac surgery ${ }^{2}$. We characterized individual inhospital complications with the use of the definitions of the Society of Thoracic Surgeons (www.sts.org). Our primary end point, defined before analysis, was a composite of serious adverse events that included in-hospital death(within 30days), myocardial infarction, asystole, ventricular tachycardia or fibrillation, tamponade, renal failure, sepsis, respiratory insufficiency, cerebralvascular accident, deep or superficial sternal-wound infection, prolonged postoperative ventilation (>72 hours), and multiorgan failure. This specific list of serious adverse events is based on the set of complications defined in the adult cardiac surgery database of the Society of Thoracic Surgeons ${ }^{6}$. There is increasing observational evidence that the risk of adverse outcomes increases incrementally with each unit of red cells/whole blood that is transfused. The increased length of hospital stay related to the number of transfused RBC/whole blood units supports a restrictive therapy in cardiac surgery. The ultimate goal of any discussion regarding the indications for blood component therapy is to maximize patient benefit and limit risk when possible. 


\section{Material and Methods}

The study will be conducted in the Dept of CTVS of SSKM Hospital, Kolkata-WB. Patients admitted for OP CABG intervention in CTVS department.

From April 2014 to July 2015. Sample size was 60 cardiac patients.

Of the 60 patients included in analysis patients receiving blood transfusion are selected for study group. The control group consists of patients will not require blood transfusion.

\section{Inclusion criteria-}

Patients with coronary artery diseases requiring coronary artery disease needing for surgical intervention with bypass grafting will be included in the study group. Patients who will under gone OPCABG will include in study.

Exclusion criteria-The following subjects will be excluded from the study

group.
- Patients with preoperative renal disease.
- Patients with preoperative anemia.
- Patients with preoperative blood transfusion.
- Patients with preoperative acute respiratory disease.
- Patients need CPB for CABG,
- Patients undergo CABG with other surgical cardiac procedures,
- Patients with CCF, Shock \& Endocarditis at the time of operation.
○ IABP (inserted before \& after operation).
- Emergency \& redo CABGs.
- Excessive perioperative blood loss (>5 units of whole blood transfused on the day of operation).
○ Re-exploration.

\section{Parameters to be studied}

The following parameters will be recorded in the subjects:

1. Record of physical Anthropometry of subjects
a. Age in years.
b. Sex
c. Height (incentimeters).

d. Weight (in kilograms).

2. Record of study parameters

The patient will be well informed about the procedure. Consent for pre and post operative transfusion. Data sheet entry of different variable parameters is made. Total no. of transfusion during hospital stay would be noted. Pre \& Post operative haemoglobin status will be assessed along with other routine parameters. Study also includes weaning off from ventilatory and ionotropic support and rate of re-intubation. Post operative rate of surgical site wound infections, complications, morbidity and mortality (within 30 days) will be observed.

\section{Study Tools}

During and after CABG, red blood cell concentrate/ whole blood transfusion requirement was assessed by repeated checking of haemoglobin level by auto analyser and i-stat EG7+analyser.

Study Techniques: Length of hospital stay will be counted from the day of surgical intervention to discharge from hospital, Total no. of red blood cell concentrate/whole blood will be transfused to maintain the hemoglobin level more than 9.0 $\mathrm{gm} / \mathrm{dl}$ during and after surgery. Arterial blood gases are done by the anaesthetist as per need. During and after OPCABG, red blood cell concentrate/ whole blood transfusion requirement will be assess by repeated checking of haemoglobin level by auto analyser/i-stat EG7+ analyser to maintain the hemoglobin level more than $9.0 \mathrm{gm} / \mathrm{dl}$ in stable and unstable patients. . Pre \&Post operative haemoglobin status will be assessed along with other routine parameters. Study also includes weaning off from ventilatory and ionotropic support and rate of reintubation.

Post-operative rate of surgical site wound infections, complications, morbidity and mortality (within 30 days) will be observed.

\section{Results}

Statistical analysis was done with standard statistical software SPSSversion20. 
Haemodynamic parameters and other normal distributed numerical variables was compared between groups by Analysis of Variance (ANOVA) followed by Tukey's Test for post hoq comparison. Within group comparison of parameters was done by repeated measured analysis of variance followed by "paired t- test" for comparison between individual time points. Catagorical variables were compared between groups by Chi-square test. All analysis was "twotailed" and "p" value less than $0.05(<0.05)$ was considered as statistically significant. From the total of 126 patients randomized chose in this study.33 did not receive any $\mathrm{RBC}$ transfusion (26.19\%) labelled them Group 1; 55 (43.65\%) patients were given 1-3 units labelled them Group 2 and $38(30.15 \%)$ received more than 3 RBC units labelled them Group 3 in the first 72 postoperative hours. Baseline characteristics of the groups are compared in Table1with mean and standard deviation and $\mathrm{p}$ value. Variables as preoperative left ventricular ejection fraction, heart failure, acute myocardial infarction, smoking hypertension, diabetes, cerebrovascular accidents and unstable angina were not associated with exposure to RBC units. Intraoperative and immediate postoperative characteristics for the RBC transfusion groups are compared in Tables 4. Most patients underwent coronary bypass artery grafting (CABG).

Patients who were exposed to blood transfusion had lower initial hematocrit and hemoglobin levels compared to patients who did not receive RBC transfusions. From 126 patients, 91 $(72.22 \%)$ patients received transfusion at the operative room and day 0(day of surgery), 58 $(46.03 \%)$ received at the first postoperative day (1PO), 19(15.08\%) at the second postoperative day (2PO) and $5(3.97 \%)$ at the third postoperative day (3PO). Analysing postoperative clinical complications, We found that patients from the high transfusion group presented a higher incidence of postoperative complications related to the composite endpoint 30-day all-cause mortality $(\mathrm{p}<0.017)$ and acute respiratory distress syndrome, and higher Re-intubation rates ( $\mathrm{p}$ $<0.004)$. Regarding infections in patients from group2 and group3 as compared with group1 we found that both groups were associated with significant $\mathrm{p}$ values \{(both deep and sternal infections) and ( $p<0.005 \&<0.020$ respectively) $\}$ It indicate blood transfusion is independent morbid factor in OPCABG patients. In our study, we compared ventilator weaning off time of the patients in different groups; we found that patients with blood transfused groups require much more time as compared to non- transfused group in all sub groups. In grop1 27(81.82\%) patients were extubated in 12-18 hrs postoperatively, and remaining $6(18.18 \%)$ patients in $18-24 \mathrm{hrs}$ postoperatively. In group2 $41(74.55 \%)$ patients extubated in $12-18 \mathrm{hrs}, 11(20 \%)$ patients extubated in 18 - $24 \mathrm{hrs}$, and only one pt took long time for weaning off from ventilator postoperatively. While in group3 only $11(28.95 \%)$ patients weanoff in 12-18 hrs, 19 (50\%) patients require 18-24 hrs time for extubation, and remaining 7 patients were extubated between 24-48 hrs postoperatively. 3 patients could not be taken off from ventilator. Similarly, in our study we found significant association between blood transfusion and postoperative inotropic support requirement for prolong time. We assessed all the groups and sub-groups for inotropic wean off time and we found that much more time required to wean off inotropic support in group 3 and group2 respectively as compared to group1, $\mathrm{p}$ values in table 7 shows significant association between no. of blood transfusion and inotropic support requirement. Higher mortality rates were directly related to blood transfusions. In our study total no. mortality was 10 . Out of these 7 deaths occur in high transfusion group i.e. group3, 2 deaths occurred in group2 and one mortality had occurred in non transfused group. Main objective our study was to found association between blood transfusion and length of stay (LOS-no. of days from day of surgery till discharge/death). In our study, Length of stay had range between 6-12 days in group1; 6-28 days in group2, and 9-45 
days in group3 with mean \& standard deviation of $7.71+/-1.67$ days in group1, $9.49+/-4.33$ days in group2 and $16.18+/-7.64$ days in group3 respectively. When we were comparing $\mathrm{p}$ values of group1, $2 \& 3$ we found highly significant values among the groups. Compare the group1 \& $2 \mathrm{p}$ value was 0.029 ; between group $1 \& 3$ and Group2 \&3 it was highly significant (0.0001). These calculations showed that no. of blood transfusions are directly responsible for increase in the length of hospitalisation of patient 1

Table1. Distribution of Patients Particulars

\begin{tabular}{|c|c|c|c|c|}
\hline $\begin{array}{c}\text { Patients } \\
\text { Particulars }\end{array}$ & $\begin{array}{l}\text { Group } 1 \\
(n=33)\end{array}$ & $\begin{array}{c}\text { Group } 2 \\
(\mathrm{n}=\mathbf{5 5})\end{array}$ & Group $3(n=38)$ & $p$ value \\
\hline Age (yrs) & $53.6+/-8.27$ & $55.05+/-8.0$ & $58.47+/-8.98$ & 0.052 \\
\hline $\operatorname{Sex}(\mathbf{F} / M)$ & $\begin{array}{c}\text { F-5 }(15.15 \%) \\
\text { M-28 } \\
(84.85 \%) \\
\end{array}$ & $\begin{array}{c}\text { F-6 }(10.9 \%) \\
\text { M-49 }(89.1 \%)\end{array}$ & $\begin{array}{c}\text { F-7 (18.42\%); } \\
\text { M-31 (81.58\%) }\end{array}$ & 0.588 \\
\hline $\begin{array}{l}\text { Weight } \\
(\mathrm{kg})\end{array}$ & $60+/-8.42$ & $60.87+/-9.89$ & $58.42+/-9.71$ & 0.417 \\
\hline $\begin{array}{l}\text { Height } \\
\text { (cm) }\end{array}$ & $161.75+/-7.45$ & $162.7+/-8.27$ & $160.57+/-8.95$ & 0.404 \\
\hline BSA & $1.62+/-0.14$ & $1.68+/-0.15$ & $1.6+/-0.15$ & 0.18 \\
\hline EF & $55.40+/-7.25$ & $51.44+/-8.22$ & $53.26+/-8.1$ & 0.326 \\
\hline
\end{tabular}

Table 2 . Distribution of associated Comorbidities

\begin{tabular}{|l|r|r|r|r|}
\hline Co-morbidities & $\begin{array}{c}\text { Group 1 } \\
(\mathrm{n}=33)\end{array}$ & Group 2 (n=55) & \multicolumn{1}{|c|}{$\begin{array}{c}\text { Group 3 } \\
(\mathrm{n}=38)\end{array}$} & p value \\
\hline Hypertension & 33 & 55 & 38 & \\
\hline $\begin{array}{l}\text { Diabetes } \\
\text { Mellitus }\end{array}$ & 12 & 24 & 21 & 0.266 \\
\hline CVA & 0 & 1 & 1 & 0.665 \\
\hline COPD & 3 & 6 & 5 & 0.863 \\
\hline Smoker & 6 & 16 & 16 & 0.088 \\
\hline $\begin{array}{l}\text { Unstable } \\
\text { Angina }\end{array}$ & 4 & 7 & 6 & 0.881 \\
\hline
\end{tabular}

Table 3 Haemoglobin Levels Pre and post operatively

\begin{tabular}{|c|c|c|c|c|}
\hline Haemoglobin & $\begin{array}{c}\text { Group 1 } \\
(\mathrm{n}=33)\end{array}$ & $\begin{array}{c}\text { Group 2 } \\
(\mathrm{n}=55)\end{array}$ & $\begin{array}{c}\text { Group 3 } \\
(\mathrm{n}=38)\end{array}$ & p value \\
\hline Pre-op & $\begin{array}{c}11.5+/- \\
9.33\end{array}$ & $\begin{array}{c}11.55+/- \\
0.938\end{array}$ & $\begin{array}{c}11.86+/- \\
1.18\end{array}$ & 0.348 \\
\hline Day 0 & $\begin{array}{c}10.13+/- \\
0.97\end{array}$ & $9.7+/-0.79$ & $9.1+/-0.89$ & 0.0001 \\
\hline Day1 & $\begin{array}{c}9.73+/- \\
0.71\end{array}$ & $9.47+/-0.53$ & $\begin{array}{c}9.36+/- \\
0.43\end{array}$ & 0.074 \\
\hline Day2 & $\begin{array}{c}9.28+/- \\
1.77\end{array}$ & $9.39+/-0.46$ & $\begin{array}{c}9.38+/- \\
0.43\end{array}$ & 0.567 \\
\hline
\end{tabular}

Table 4 Blood transfusions

\begin{tabular}{|c|c|c|c|}
\hline $\begin{array}{c}\text { Total } \\
\text { Blood } \\
\text { Units }\end{array}$ & $\begin{array}{c}\text { Group } \\
\mathbf{1} \\
(\mathbf{n}=\mathbf{3 3})\end{array}$ & $\begin{array}{c}\text { Group } \\
\mathbf{2} \\
(\mathbf{n}=\mathbf{5 5})\end{array}$ & $\begin{array}{c}\text { Group } \\
\mathbf{3} \\
(\mathbf{n}=\mathbf{3 8})\end{array}$ \\
\hline $\mathbf{1}$ & $\mathbf{0}$ & $\mathbf{1 4}$ & $\mathbf{0}$ \\
\hline $\mathbf{2}$ & $\mathbf{0}$ & $\mathbf{2 2}$ & $\mathbf{0}$ \\
\hline $\mathbf{3}$ & $\mathbf{0}$ & $\mathbf{1 9}$ & $\mathbf{0}$ \\
\hline $\mathbf{4}$ & $\mathbf{0}$ & $\mathbf{0}$ & $\mathbf{1 1}$ \\
\hline $\mathbf{5}$ & $\mathbf{0}$ & $\mathbf{0}$ & $\mathbf{1 8}$ \\
\hline $\mathbf{6}$ & $\mathbf{0}$ & $\mathbf{0}$ & $\mathbf{4}$ \\
\hline 7 & $\mathbf{0}$ & $\mathbf{0}$ & $\mathbf{4}$ \\
\hline $\mathbf{8}$ & $\mathbf{0}$ & $\mathbf{0}$ & $\mathbf{0}$ \\
\hline $\mathbf{9}$ & $\mathbf{0}$ & $\mathbf{0}$ & $\mathbf{1}$ \\
\hline
\end{tabular}

\begin{tabular}{c|c|c|c|}
\hline $\begin{array}{c}\text { No. of Blood } \\
\text { Transfusions } \\
\text { Day 0 }\end{array}$ & $\begin{array}{c}\text { Group } \\
1 \\
(\mathbf{1}=33)\end{array}$ & $\begin{array}{c}\text { Group } \\
2 \\
(\mathbf{n}=55)\end{array}$ & $\begin{array}{c}\text { Group } \\
3 \\
(\mathbf{n}=38)\end{array}$ \\
\hline NO & 0 & 2 & 0 \\
\hline 1 & 0 & 26 & 0 \\
\hline 2 & 0 & 22 & 5 \\
\hline 3 & 0 & 5 & 18 \\
\hline 4 & 0 & 0 & 13 \\
\hline 5 & 0 & 0 & 2
\end{tabular}

\begin{tabular}{|c|c|c|c|}
\hline $\begin{array}{c}\text { No. of Blood } \\
\text { Transfusions } \\
\text { Day 1 }\end{array}$ & $\begin{array}{c}\text { Group } \\
1 \\
(\mathrm{n}=33)\end{array}$ & $\begin{array}{c}\text { Group } \\
2 \\
(\mathrm{n}=55)\end{array}$ & $\begin{array}{c}\text { Group } \\
\mathbf{3} \\
(\mathrm{n}=38)\end{array}$ \\
\hline NO & 0 & 30 & 5 \\
\hline 1 & 0 & 23 & 22 \\
\hline 2 & 0 & 2 & 10 \\
\hline 3 & 0 & 0 & 1 \\
\hline
\end{tabular}

\begin{tabular}{|c|c|c|c|}
\hline $\begin{array}{c}\text { No. of Blood } \\
\text { Transfusions } \\
\text { Day 2 }\end{array}$ & $\begin{array}{c}\text { Group } \\
\mathbf{1} \\
(\mathrm{n}=33)\end{array}$ & $\begin{array}{c}\text { Group } \\
2 \\
(\mathrm{n}=55)\end{array}$ & $\begin{array}{c}\text { Group } \\
\mathbf{3} \\
(\mathrm{n}=38)\end{array}$ \\
\hline NO & 0 & 53 & 21 \\
\hline 1 & 0 & 2 & 16 \\
\hline 2 & 0 & 0 & 1 \\
\hline
\end{tabular}




\begin{tabular}{|c|c|c|c|}
\hline $\begin{array}{c}\text { No. of Blood } \\
\text { Transfusions } \\
\text { Day 3 }\end{array}$ & $\begin{array}{c}\text { Group } \\
\mathbf{1} \\
(\mathbf{n}=\mathbf{3 3})\end{array}$ & $\begin{array}{c}\text { Group } \\
\mathbf{2} \\
(\mathbf{n}=\mathbf{5 5})\end{array}$ & $\begin{array}{c}\text { Group } \\
\mathbf{3} \\
(\mathbf{n}=\mathbf{3 8})\end{array}$ \\
\hline NO & 0 & 54 & 34 \\
\hline 1 & 0 & 1 & 3 \\
\hline 2 & 0 & 0 & 1 \\
\hline
\end{tabular}

Table 5.Post operative complications

\begin{tabular}{|c|c|c|c|c|}
\hline $\begin{array}{c}\text { Post-op } \\
\text { Complications }\end{array}$ & $\begin{array}{c}\text { Group 1 } \\
(\mathrm{n}=33)\end{array}$ & $\begin{array}{c}\text { Group 2 } \\
(\mathrm{n}=55)\end{array}$ & $\begin{array}{c}\text { Group 3 } \\
(\mathrm{n}=38)\end{array}$ & p value \\
\hline ARDS & $2(6.06 \%)$ & $\begin{array}{c}8 \\
(14.55 \%)\end{array}$ & $9(23.68 \%)$ & 0.116 \\
\hline ARF & $2(6.06 \%)$ & $3(5.45 \%)$ & $3(7.89 \%)$ & 0.891 \\
\hline CHF & $2(6.06 \%)$ & $\begin{array}{c}8 \\
(14.55 \%)\end{array}$ & $9(23.68 \%)$ & 0.116 \\
\hline REINTUBATION & $1(3.03 \%)$ & $4(7.27 \%)$ & $\begin{array}{c}10 \\
(26.37 \%)\end{array}$ & 0.004 \\
\hline
\end{tabular}

Table 6 Ventilator wean off(hrs)

\begin{tabular}{|c|c|c|c|c|}
\hline $\begin{array}{c}\text { Ventilator } \\
\text { Wean off } \\
\text { (hrs) }\end{array}$ & $\begin{array}{c}\text { Group 1 } \\
(\mathbf{n}=\mathbf{3 3})\end{array}$ & $\begin{array}{c}\text { Group 2 } \\
(\mathbf{n}=\mathbf{5 5})\end{array}$ & $\begin{array}{c}\text { Group 3 } \\
(\mathbf{n}=\mathbf{3 8})\end{array}$ & p value \\
\hline $\mathbf{1 2 - 1 8}$ hrs & 27 & 41 & 11 & 0.0001 \\
\hline $\mathbf{1 8 - 2 4}$ hrs & 6 & 11 & 19 & 0.002 \\
\hline $\mathbf{2 4 - 4 8}$ hrs & 0 & 1 & 7 & 0.001 \\
\hline $\mathbf{4 8 - 7 2 ~ h r s ~}$ & 0 & 0 & 0 & NA \\
\hline Not wean off & 0 & 2 & 1 & NA \\
\hline
\end{tabular}

Table 7 Inotropic wean off

\begin{tabular}{|c|c|c|c|c|}
\hline $\begin{array}{c}\text { Inotropic } \\
\text { Wean off } \\
(\text { hrs })\end{array}$ & $\begin{array}{c}\text { Group 1 } \\
(\mathrm{n}=33)\end{array}$ & $\begin{array}{c}\text { Group 2 } \\
(\mathrm{n}=55)\end{array}$ & $\begin{array}{c}\text { Group 3 } \\
(\mathrm{n}=38)\end{array}$ & $\begin{array}{c}\text { p } \\
\text { Value }\end{array}$ \\
\hline$<6$ hrs & $\begin{array}{c}13 \\
(39.39 \%)\end{array}$ & $11(20 \%)$ & $\begin{array}{c}6 \\
(15.79 \%)\end{array}$ & 0.045 \\
\hline $6-12 \mathrm{hrs}$ & $\begin{array}{c}4 \\
(12.12 \%)\end{array}$ & $\begin{array}{c}10 \\
(18.18 \%)\end{array}$ & $\begin{array}{c}4 \\
(10.53 \%)\end{array}$ & 0.536 \\
\hline $12-18 \mathrm{hrs}$ & $\begin{array}{c}10 \\
(30.30 \%)\end{array}$ & $\begin{array}{c}6 \\
(10.91 \%)\end{array}$ & $0(0 \%)$ & 0.001 \\
\hline $18-24 \mathrm{hrs}$ & $\begin{array}{c}3 \\
(9.09 \%)\end{array}$ & $\begin{array}{c}8 \\
(14.55 \%)\end{array}$ & $\begin{array}{c}1 \\
(2.63 \%)\end{array}$ & 0.156 \\
\hline $24-48 \mathrm{hrs}$ & $\begin{array}{c}1 \\
(3.03 \%)\end{array}$ & $\begin{array}{c}12 \\
(21.82 \%)\end{array}$ & $\begin{array}{c}9 \\
(23.68 \%)\end{array}$ & 0.039 \\
\hline $48-72 \mathrm{hrs}$ & $\begin{array}{c}1 \\
(3.03 \%)\end{array}$ & $\begin{array}{c}6 \\
(10.91 \%)\end{array}$ & $\begin{array}{c}18 \\
(47.37 \%)\end{array}$ & 0.0001 \\
\hline
\end{tabular}

Table 8 Infection

\begin{tabular}{|c|c|c|c|c|}
\hline Infection & $\begin{array}{c}\text { Group 1 } \\
(\mathrm{n}=33)\end{array}$ & $\begin{array}{c}\text { Group } \\
2 \\
(\mathrm{n}=55)\end{array}$ & $\begin{array}{c}\text { Group 3 } \\
(\mathrm{n}=38)\end{array}$ & $\begin{array}{c}\mathrm{p} \\
\text { Value }\end{array}$ \\
\hline Superficial & $\begin{array}{c}4 \\
(12.12 \%)\end{array}$ & $\begin{array}{c}6 \\
(10.9 \%)\end{array}$ & $\begin{array}{c}8 \\
(21.08 \%)\end{array}$ & 0.357 \\
\hline Deep & $\begin{array}{c}4 \\
(12.12 \%)\end{array}$ & $\begin{array}{c}2 \\
(3.64 \%)\end{array}$ & $\begin{array}{c}10 \\
(26.32 \%)\end{array}$ & 0.05 \\
\hline Sternal & $0(0 \%)$ & $\begin{array}{c}4 \\
(7.27 \%)\end{array}$ & $\begin{array}{c}7 \\
(18.42 \%)\end{array}$ & 0.02 \\
\hline
\end{tabular}

Table 9 Mortality

\begin{tabular}{|c|c|c|c|c|}
\hline MORTALITY & $\begin{array}{c}\text { Group } \\
1 \\
(\mathrm{n}=33)\end{array}$ & $\begin{array}{c}\text { Group } \\
2 \\
(\mathrm{n}=55)\end{array}$ & $\begin{array}{c}\text { Group 3 } \\
(\mathrm{n}=38)\end{array}$ & $\begin{array}{c}\mathrm{p} \\
\text { Value }\end{array}$ \\
\hline Mortality & $\begin{array}{c}1 \\
(3.03 \%)\end{array}$ & $\begin{array}{c}2 \\
(3.64 \%)\end{array}$ & $\begin{array}{c}7 \\
(18.42 \%)\end{array}$ & 0.017 \\
\hline
\end{tabular}

Table 10.Length of stay

\begin{tabular}{|c|c|c|c|c|}
\hline $\begin{array}{c}\text { Length of } \\
\text { Stay }\end{array}$ & $\begin{array}{c}\text { Group } \\
1 \\
(\mathrm{n}=33)\end{array}$ & $\begin{array}{c}\text { Group } \\
2 \\
(\mathrm{n}=55)\end{array}$ & $\begin{array}{c}\text { Group } \\
3 \\
(\mathrm{n}=38)\end{array}$ & $\begin{array}{c}\mathrm{p} \\
\text { Value }\end{array}$ \\
\hline \multirow{3}{*}{ LOS(days) } & $\begin{array}{c}7.71 \\
+/-\end{array}$ & $\begin{array}{c}9.49+/- \\
4.33\end{array}$ & $\begin{array}{c}16.18 \\
+/-7.64\end{array}$ & 0.0001 \\
& $(1.67)$ & & & \\
\hline
\end{tabular}

\section{Conclusion}

In conclusion, our data for OPCABG shows that patients who received transfusion required significantly longer ventilator support time, longer inotropic support time, increase rates of reintubation. All these factors combine to increase morbidity and delays patient's recovery process. In our study, we found that blood transfusions increase the rates of superficial and deep sternal wound infections, increases hospital stay of patient. All above factors contributing to increase mortality in higher blood transfused patients. They also had significantly higher incidence of the 30day mortality. We found that LOS increased with the number of units of blood transfused. Thus we suggest that for better patient outcome, early 
recovery, full utilisation of scarce resources, decrease unnecessary economic burden to community, we should approach toward the restricted blood transfusions and pre-op management of risk factors.

\section{References}

1. Gavin J. Murphy; Barnaby C. Reeves,; Chris A. Rogers,; Syed I.A. Rizvi,; Lucy Culliford,; Gianni D.: Increased Mortality, Postoperative Morbidity, and Cost After Red Blood Cell Transfusion in Patients Having Cardiac Surgery. Circulation. 2007; 116: 2544-2552.

2. Filomena RBG Galas1, Juliano $P$ Almeida1, Julia T Fukushima1, Eduardo A Osawa1, Rosana E Nakamura1, Carolina MPDC Silva1, Elisângela Pinto Marinho de Almeida1, Jose Otavio Costa Auler Jr1, Jean-Louis Vincent2 and Ludhmila A Hajjar1: Blood transfusion in cardiac surgery is a risk factor for increased hospital length of stay in adult patients. Journal of Cardiothoracic Surgery 2013, 8:54.

3. Bernard AC, Davenport DL, Chang PK, Vaughan TB, Zwischenberger JB.: Intraoperative transfusion of $1 \mathrm{U}$ to $2 \mathrm{U}$ packed red blood cells is associated with increased 30-day mortality, surgical-site infection, pneumonia, and sepsis in general surgery patients. J Am CollSurg 2009 May;208(5):931-7, 937.e1-2; discussion 938-9. Epub 2009 Mar 26.

4. Surgenor SD, Kramer RS, Olmstead EM, Ross CS, Sellke FW, Likosky DS, Marrin CA, Helm RE Jr, Leavitt BJ, Morton JR, Charlesworth DC, Clough RA, Hernandez $\mathrm{F}$, Frumiento $\mathrm{C}$, Benak A, DioData $\mathrm{C}$, O'Connor GT: Northern New England Cardiovascular Disease Study Group. The association of perioperative red blood cell transfusions and decreased long-term survival after cardiac surgery. Anesth Analg 2009, 108:1741-1746.
5. Santiago Ramón Leal-Noval, ;María Dolores Rincón-Ferrari, ; Andrés GarcíaCuriel,; Angel Herruzo-Avilés,; Pedro Camacho-Laraña, José GarnachoMontero,; Rosario Amaya-Villar,: Transfusion of Blood Components and Postoperative Infection in Patients Undergoing Cardiac Surgery. Clinical Investigations | SURGERY | May 2001, Vol 119, No. 5.

6. Colleen Gorman Koch, Liang Li, Daniel I. Sessler, Priscilla Figueroa, Gerald A. Hoeltge, Tomislav Mihaljevic, and Eugene H. Blackstone,: Duration of Red-Cell Storage and Complications after Cardiac Surgery. N Engl J Med 2008; 358:12291239.

7. Rajesh Chand Arya, GS Wander, and Pankaj Gupta Blood component therapy: Which, when and how much. J Anaesthesiol Clin Pharmacol. 2011 AprJun; 27(2): 278-284.

8. Perioperative Blood Transfusion and Blood Conservation in Cardiac Surgery: The Society of Thoracic Surgeons and The Society of Cardiovascular Anesthesiologists Clinical Practice Guideline; Annals of Thoracic Surgery, Volume 83, Issue 5, Supplement, Pages S27-S86, May 2007.

9. Jay Alan E. Junio, MD; Reynante Gamponia, MD; Post-operative Outcomes of CABG Patients Given Blood Transfusion Based on Society of Thoracic Surgeons Guidelines on Blood Transfusion Phil Heart Center J 2012; 16(2):47-54.

10. Carson, JL; Grossman, BJ, Kleinman, S, Tinmouth, AT, Marques, MB, Fung, MK, Holcomb, JB, Illoh, O, Kaplan, LJ, Katz, LM, Rao, SV, Roback, JD, Shander, A, Tobian, AA, Weinstein, R, Swinton McLaughlin, LG, Djulbegovic, B, for the Clinical Transfusion Medicine Committee of the, AABB (Mar 26, 2012). "Red Blood Cell Transfusion: A Clinical Practice Guideline From the AABB". Annals of 
internal medicine. doi:10.1059/0003-4819156-12-201206190-00429

11. Murphy GJ, Reeves BC, Rogers CA, Rizvi SI, Culliford L, et al. (2007), Increased mortality, postoperative morbidity, and cost after red blood cell transfusion in patients having cardiac surgery. Circulation 116: 2544-2552, 2007.

12. Hare GM, Baker JE, Mazer CD (2009), Perioperative management of acute and chronic anemia: has the pendulum swung too far? Can J Anaesth 56: 183-189,2009.

13. Habib RH, Zacharias A, Schwann TA, Riordan CJ, Durham SJ, et al. (2003) Adverse effects of low hematocrit during cardiopulmonary bypass in the adult: should current practice be changed? J Thorac Cardiovasc Surg 125: 14381450,2003.

14. Karkouti K, Djaiani G, Borger MA, Beattie WS, Fedorko L, et al. (2005) Low hematocrit during cardiopulmonary bypass is associated with increased risk of perioperative stroke in cardiac surgery. Ann Thorac Surg 80: 1381-1387,2005.

15. Karkouti K, Wijeysundera DN, Beattie WS, Reducing Bleeding in Cardiac Surgery I (2008) Risk associated with preoperative anemia in cardiac surgery: a multicenter cohort study. Circulation 117: 478-484,2008.

16. Kulier A, Levin J, Moser R, RumpoldSeitlinger G, Tudor IC, et al. (2007) Impact of preoperative anemia on outcome in patients undergoing coronary artery bypass graft surgery. Circulation 116: 471479,2007.

17. Swaminathan M, Phillips-Bute BG, Conlon PJ, Smith PK, Newman MF, et al. (2003) The association of lowest hematocrit during cardiopulmonary bypass with acute renal injury after coronary artery bypass surgery. Ann Thorac Surg 76: 784-791,2003.
18. DeFoe GR, Ross CS, Olmstead EM, Surgenor SD, Fillinger MP, et al. (2001) Lowest hematocrit on bypass and adverse outcomes associated with coronary artery bypass grafting. Northern New England Cardiovascular Disease Study Group. Ann ThoracS urg 71: 769-776,2001.

19. Zubair, AC (2010). "Clinical impact of blood storage lesions". American journal of hematology 85 (2):117-22,2010.

20. Tsai AG, Cabrales $P$, Intaglietta $M$. Microvascular perfusion upon exchange transfusion with stored red blood cells in normovolemic anemic conditions. Transfusion. 2004;44:1626 -1634.

21. Raat NJ, Verhoeven AJ, Mik EG, Gouwerok CW, Verhaar R, Goedhart PT, de Korte D, Ince C. The effect of storage time of human red cells on intestinal microcirculatory oxygenation in a rat isovolemic exchange model. Crit Care Med. 2005;33:39-45.

22. Hovav T, Yedgar S, Manny N, Barshtein G. Alteration of red cell aggregability and shape during blood storage. Transfusion. 1999;39: 277-281.

23. d'Almeida MS, Jagger J, Duggan M, White M, Ellis C, Chin-Yee IH. A comparison of biochemical and functional alterations of rat and human erythrocytes stored in CPDA-1 for 29 days: implications for animal models of transfusion. Transfus Med. 2000;10:291303.

24. Crawford JH, Isbell TS, Huang Z, Shiva S, Chacko BK, Schechter AN, Darley-Usmar VM, Kerby JD, Lang JD Jr, Kraus D, Ho C, Gladwin MT, Patel RP. Hypoxia, red blood cells, and nitrite regulate NOdependent hypoxic vasodilation. Blood. 2006; 107:566 -574.

25. Simak J, Gelderman MP. Cell membrane microparticles in blood and blood products: potentially pathogenic agents 
and diagnostic markers. Trans Med Rev. 2006;20:1-26.

26. Silliman CC, Voelkel NF, Allard JD, Elzi DJ, Tuder RM, Johnson JL, Ambruso DR. Plasma and lipids from stored packed red blood cells cause acute lung injury in an animal model. J Clin Invest. 1998;101: $1458-1467$.

27. Fransen E, Maessen J, Dentener M, Senden N, Buurman W. Impact of blood transfusions on inflammatory mediator release in patients undergoing cardiac surgery. Chest. 1999;116:1233-1239.

28. M. Kamran Athar,1Nitin Puri,2 and David R. Gerber3,4,5. Anemia and Blood Transfusions in Critically Ill Patients Journal of Blood Transfusion Volume 2012 (2012), Article ID 629204, 7 pages.

29. Wang, T; Luo, L; Huang, H; Yu, J; Pan, C; Cai, X; Hu, B; Yin, X (May 2014). "Perioperative blood transfusion is associated with worse clinical outcomes in resected lung cancer.". The Annals of $\begin{array}{lll}\text { thoracic surgery } 97 & \text { (5):182737. }\end{array}$ doi:10.1016/j.athoracsur.2013.12.044.

PMID 24674755.

30. Churchhouse, AM; Mathews, TJ; McBride, OM; Dunning, J (Jan 2012). "Does blood transfusion increase the chance of recurrence in patients undergoing surgery for lung cancer?". Interactive cardiovascular and thoracic surgery 14(1):85,90. doi:10.1093/icvts/ivr025.PMID22108935

31. Corwin HL, Gettinger A, Pearl RG, Fink MP, Levy MM, Abraham E, MacIntyre NR, Shabot MM, Duh MS, Shapiro MJ. The CRIT Study: Anemia and blood transfusion in the critically ill--current clinical practice in the United States. Crit Care Med. 2004 Jan;32(1):39-52.

32. Hajjar LA, Vincent JL, Galas FR, Nakamura RE, Silva CM, Santos MH, et al. Transfusion requirements after cardiac surgery: the TRACS randomized controlled trial. JAMA. 2010;304 (14):1559-67.

33. Leal-Noval SR, Rincón-Ferrari MD, García-Curiel A, Herruzo-Avilés A, Camacho-Laraña P, Garnacho-Montero J, et al. Transfusion of blood components and postoperative infection in patients undergoing cardiac surgery. Chest. 2001;119(5):1461-8.

34. Engoren MC, Habib RH, Zacharias A, Schwann TA, Riordan CJ, Durham SJ. Effect of blood transfusion on long-term survival after cardiac operation. Ann Thorac Surg. 2002;74(4):1180-6.

35. B. F. Mazza, F. R. Machado, D. D. Mazza, and V. Hassmann, "Evaluation of blood transfusion effects on mixed venous oxygen saturation and lactate levels in patients with SIRS/sepsis," Clinics, vol. 60, no. 4, 2005, pp. 311-316,

36. Ferraris VA, Ferraris SP, Saha SP, et al. Perioperative blood transfusion and blood conservation in cardiac surgery: The Society of Thoracic Surgeons and the Society of Cardiovascular Anesthesiologists clinical practice guideline. Ann Thorac Surg 2007;83(Suppl):27- 86.

37. Barnum JL, Sistino JJ. Renal dysfunction in cardiac surgery: identifying potential risk factors. Perfusion 2009;24:139-42.

38. Bainbridge D, Martin J, Cheng D. Off pump coronary artery bypass graft surgery versus conventional coronary artery bypass graft surgery: a systematic review of the literature. Semin Cardiothorac Vasc Anesth 2005;9:105-11.

39. Pandey R, Grayson AD, Pullan DM, Fabri BM, Dihmis WC. Total arterial revascularisation: effect of avoiding cardiopulmonary bypass on in-hospital mortality and morbidity in a propensitymatched cohort. Eur J CardiothoracSurg 2005;27:94-8.

40. Koch CG, Li L, Duncan AI, Mihaljevic T, Cosgrove DM, Loop FD, Starr NJ, 
Blackstone EH: Morbidity and mortality risk associated with red blood cell and blood-component transfusion in isolated coronary artery bypass grafting. Crit Care Med 2006, 34:1608-1616.

41. Murphy GJ, Reeves BC, Rogers CA, Rizvi SI, Culliford L, Angelini GD: Increased mortality, postoperative morbidity, and cost after red blood cell transfusion in patients having cardiac surgery. Circulation 2007, 116:2544-2552.

42. Goodnough, LT, Despostis, GJ, Hogue, $\mathrm{CW}$, et al On the need for improved transfusion indicators in cardiac surgery. Ann Thorac Surg1995;60,473-480.

43. Camila de Christo DornelesI; Luiz Carlos BodaneseII; João Carlos Vieira da Costa GuaragnaIII; Fabrício Edler MacagnanIV; Juliano CéCoelhoV; Anibal Pires BorgesVI; Marco Antonio GoldaniVII; João Batista Petracco VIII; The impact of blood transfusion on morbidity and mortality after cardiac surgery; Rev Bras Cir Cardiovasc vol.26 no.2 São José do Rio Preto Apr./June 2011.

44. Vamvakas EC1, Carven JH. Allogeneic blood transfusion, hospital charges, and length of hospitalization: a study of 487 consecutive patients undergoing colorectal cancer resection. Arch Pathol Lab Med. 1998 Feb;122(2):145-51. 